\author{
Applicable Analysis And Discrete Mathematics \\ available online at http://pefmath.etf.rs
}

Appl. Anal. Discrete Math. 6 (2012), 46-62.

doi:10.2298/AADM111230001A

\title{
ATOMS AND CLIQUE SEPARATORS IN GRAPH PRODUCTS
}

\author{
Bijo S. Anand, Kannan Balakrishnan, Manoj Changat, Iztok Peterin
}

We describe in the present work all minimal clique separators of the four standard products-Cartesian, strong, direct, and lexicographic-as well as all maximal atoms of the Cartesian, strong and lexicographic product, while we only partially describe maximal atoms of direct products. Typically, a product has no clique separator and so the product is a maximal atom.

\section{INTRODUCTION AND PRELIMINARIES}

A clique separator of a graph $G$ with $k$ components is a clique (a subgraph consisting of pairwise adjacent vertices) in $G$ whose removal disconnects the graph into more than $k$ components. Clearly every clique separator is a subgraph of just one component of $G$. A clique separator of $G$ is said to be a minimal clique separator if it does not contain any other clique separator of $G$. A connected induced subgraph of $G$ that contains no clique separators is called an atom of $G$. A maximal atom of $G$ is an atom of $G$ which is not contained in any other atom of $G$.

Decomposing a graph into atoms and clique separators is a very important problem, algorithmically or otherwise, because, many hard graph problems like finding a maximum size clique can be optimized by first decomposing the graph into smaller clique separator-free graphs. This decomposition also preserves induced cycles, holes, and anti-holes in the graph. Decomposition by clique separators of a graph was first obtained by TARJAN [16], where he designed an $O(m n)$ algorithm. Tarjan's decomposition may not produce a unique decomposition of the graph. He left open the problem of such a unique decomposition. An algorithm yielding

2010 Mathematics Subject Classification. 05C69, 05C76.

Keywords and Phrases. Clique separator, atom, graph products. 
a unique decomposition containing all the atoms of the graph was obtained by LEIMER in [7]. A review on the topic can be found in [4].

One of first non-algorithmic results on clique separators goes even further back to DIRAC in 1961 [6], where he characterized chordal graphs using clique separators. The atoms and clique separators of graphs also play an important role in one type of graph convexity, namely the induced path convexity. The induced path convex set or $J$-convex set in a connected graph $G=(V, E)$ is a subset $W$ of $V$ which contains vertices on all induced paths between all pairs of vertices in $W$. The induced path convexity is a well-studied convexity in graphs as can be seen from the papers $[\mathbf{9}, \mathbf{8}]$ and the references therein. Note that a characterization of the convex hulls of induced path convex sets in a graph $G=(V, E)$ due to Duchet [9] involves clique separators. Using this characterization and an application of the decomposition algorithm [7], an algorithm for computing the induced path convex hull of a vertex subset of $G$ is presented in [2].

Various graph products have been investigated in the last few decades and a rich theory involving the structure and recognition of classes of these graphs has emerged, cf. the new book [11]. The most studied graph products are the Cartesian product, the strong product, the direct product, and the lexicographic product which are also called standard products. The other standard approach to graph products is to deduce properties of a product with respect to its factors. See a short collection of such results in $[\mathbf{3}, \mathbf{1 0}, \mathbf{1 2}, \mathbf{1 3}, \mathbf{1 8}]$. We wish to obtain results of that type for minimal clique separators and maximal atoms for all standard products. Recently this was done for the minimal vertex separators of the Cartesian product in [14], of the strong product in [15] and was discussed for the direct prodcut in [5]. A characterization of the induced path (as well as the geodesic and the Steiner) convex sets in lexicographic product was presented in [1].

In this paper, we study the atoms and clique separators of all standard products. For this we completely describe minimal clique separating sets of all four products as well as maximal atoms of the Cartesian, the lexicographic, and the strong product, while for the direct product this task is done partially. These structural characterization enable us to apply the decomposition algorithm from [4] to obtain fast decompositions in lexicographic and strong product in comparison with applying the algorithm directly. We estimate the time complexity of such a procedure. The paper is organized as follows. In the remainder of this section we define all four standard products and give some of their basic properties. The next sections are then devoted to minimal clique separators and maximal atoms of the Cartesian, the lexicographic, the strong, and the direct product, respectively. We end with a section on the decomposition algorithms.

For all four products of (simple) graphs $G$ and $H$ the vertex set of the product is $V(G) \times V(H)$. Their edge sets are defined as follows. In the Cartesian product $G \square H$ two vertices are adjacent if they are adjacent in one coordinate and equal in the other. In the direct product $G \times H$ two vertices are adjacent if they are adjacent in both coordinates. The edge set $E(G \otimes H)$ of the strong product $G \otimes H$ is the union of $E(G \square H)$ and $E(G \times H)$. Finally, two vertices $(g, h)$ and $\left(g^{\prime}, h^{\prime}\right)$ 
are adjacent in the lexicographic product $G \circ H$ (also $G[H]$ ) either if $g g^{\prime} \in E(G)$ or if $g=g^{\prime}$ and $h h^{\prime} \in E(H)$. For $* \in\{\square, \bigotimes, \times, \circ\}$ we call the product $G * H$ nontrivial, if both $G$ and $H$ have at least two vertices. For $h \in V(H), g \in V(G)$, and $* \in\{\square, \bigotimes, \times, \circ\}$, denote by $G^{h}=\{(g, h) \in G * H: g \in V(G)\}$ a $G$ layer in $G * H$, and denote by ${ }^{g} H=\{(g, h) \in G * H: h \in V(H)\}$ an $H$ layer in $G * H$. Note that the subgraph of $G * H$ induced on $G^{h}$ is isomorphic to $G$ and the subgraph of $G * H$ induced on ${ }^{g} H$ is isomorphic to $H$ for $* \in\{\square, \bigotimes, \circ\}$. On the other hand there are no edges between vertices of $G^{h}$ and between vertices of ${ }^{g} H$ in $G \times H$. Note also that all four products are associative and only first three are commutative while the lexicographic product is not, cf. [11]. The map $p_{G}: V(G * H) \rightarrow V(G)$ defined with $p_{G}((g, h))=g$ is called a projection map onto $G$ for $* \in\{\square, \bigotimes, \times, \circ\}$. Similarly we can define the projection map onto $H$.

Let $S \subset V(G)$. With $\langle S\rangle$ we denote the subgraph of $G$ induced by $S$. We will also use for a graph $G$ the standard notations $N^{G}(g)$ for the open neighborhood $\left\{g^{\prime}: g g^{\prime} \in E(G)\right\}, N^{G}[g]$ for the closed neighborhood $N^{G}(g) \cup\{g\}$, and $S_{k}^{G}(g)$ for the $k$-th sphere $\left\{g^{\prime}: d_{G}\left(g, g^{\prime}\right)=k\right\}$.

\section{THE CARTESIAN PRODUCT}

In this section we give a short discussion on the (minimal) clique separator and (maximal) atoms in the Cartesian product. As we will see there are no clique separators in most Cartesian product graphs and thus most of them do not contain any proper atoms. Note that for general minimal vertex separators this is not the case cf. $[\mathbf{1 4}]$.

Let $(g, h)$ and $\left(g^{\prime}, h^{\prime}\right)$ be two vertices that are not in the same $(G$ or $H)$ layer, that is $g \neq g^{\prime}$ and $h \neq h^{\prime}$. They are clearly not adjacent and thus not in the same clique. Hence every clique of $G \square H$ must be contained in one layer, say $G^{h}$. Furthermore, a subset $A$ of $G^{h}$ is clearly a separator of $G \square H$ for connected graphs $G$ and $H$ if and only if $A=G^{h}$ and $h$ is a cut vertex of $H$. Since we are interested in clique separators only, this implies that $G^{h}$ must induce a complete graph and hence $G$ is also a complete graph. The following proposition is then clear.

Proposition 2.1. The nontrivial Cartesian product $G \square H$ of connected graphs $G$ and $H$ has a (minimal) clique separator $K$ if and only if either $K=\left\langle G^{h}\right\rangle$ where $G$ is complete and $h$ is a cut vertex of $H$ or $K=\left\langle{ }^{g} H\right\rangle$ where $H$ is complete and $g$ is a cut vertex of $G$.

Let $g_{1}, \ldots, g_{k}$ be cut vertices of $G$. For $g_{1}$ and a component $C_{i}, i=1, \ldots, i_{g_{1}}$ of $G-\left\{g_{1}\right\}$, we denote by $C_{i}^{g_{1}}=\left\langle C_{i} \cup\left\{g_{1}\right\}\right\rangle$ and by $G_{1}=C_{1}^{g_{1}} \cup \cdots \cup C_{i_{g_{1}}}^{g_{1}}$. We continue with the same procedure with $g_{j}$ on $G_{j-1}$ for every $j>1$. At the end we obtain a graph $G^{+}=G_{k}$ that is not connected whenever $k \geq 1$. In particular note that for two adjacent cut vertices $g_{i}$ and $g_{j}$, the edge $g_{i} g_{j}$ is a component of $G_{k}$. Also $G^{+}$has no cut vertices and every vertex of $V(G)-\left\{g_{1}, \ldots, g_{k}\right\}$ is in exactly one component of $G^{+}$. If $G$ has no cut vertices we simply write $G^{+}=G$.

Theorem 2.2. The nontrivial Cartesian product $G \square H$ of connected graphs $G$ and $H$ has a maximal atom $A$ if and only if either 
(i) $A=G \square C_{H}$ where $G$ is complete and $C_{H}$ a component of $H^{+}$, or

(ii) $A=C_{G} \square H$ where $H$ is complete and $C_{G}$ a component of $G^{+}$, or

(iii) $A=G \square H$ if $G$ and $H$ are not complete.

Proof. Since $G \square H$ is nontrivial, both $G$ and $H$ have at least two vertices. If $G$ and $H$ are not complete graphs, $A=G \square H$ has no clique separators by Proposition 2.1 and hence $A$ is an atom. If $H$ is a complete graph and $C_{G}$ a component of $G^{+}$, then $C_{G}$ has no cut vertices. By Proposition 2.1, $A=C_{G} \square H$ has no clique separators and hence $A$ is an atom. Moreover, if $A \subset A^{\prime}$ there exists $(g, h) \in A^{\prime}-A$, which is a neighbor of $\left(g^{\prime}, h\right) \in A$. Clearly $A^{\prime}$ is not an atom, since $\left\{g^{\prime}\right\} \times H$ is a clique separator of $A^{\prime}$. Hence $A$ is a maximal atom. The same can be concluded for $A=G \square C_{H}$ when $G$ is complete and $C_{H}$ a component of $H^{+}$.

Conversely, note that conditions (i), (ii), and (iii) have pairwise empty intersections. Now, let $A$ be a maximal atom of $G \square H$. If $G$ and $H$ are not complete, $A=G \square H$ contains no clique separators by Proposition 2.1 and (iii) follows. So let $H$ be a complete graph. If $p_{G}(A)$ contains a cut vertex $g$ of $\left\langle p_{G}(A)\right\rangle$, then ${ }^{g} H \cap A$ is a clique separator by Proposition 2.1 again, which is a contradiction. Hence $p_{G}(A)$ has no cut vertices in $\left\langle p_{G}(A)\right\rangle$. On the other hand, maximal subgraphs of $G$ without cut vertices are clearly isomorphic to components of $G^{+}$. Let $C$ be a component of $G^{+}$with $\left|V(C) \cap p_{G}(A)\right| \geq 2$. Note that such a component exists, since $\left|p_{G}(A)\right| \geq 2$ by maximality of $A$. By Proposition 2.1, $C \square H$ has no clique separators and $C \square H \subseteq A$ by the maximality of $A$ again. But then $A=C \square H$, otherwise we get the same contradiction again and (ii) follows. By symmetry, (i) follows if $G$ is a complete graph.

\section{THE LEXICOGRAPHIC PRODUCT}

We begin with a necessary and sufficient condition for the existence of clique separators in the lexicographic product $G \circ H$.

Proposition 3.3. Let $G$ be a connected graph and $H$ a graph. The nontrivial lexicographic product $G \circ H$ has a clique separator $K^{\prime}$ if and only if $G$ has a clique separator $K$ and $H$ is a complete graph.

Proof. Suppose $G$ has a clique separator $K$ and $H$ is complete. Clearly $K^{\prime}=K \circ H$ is a clique in $G \circ H$. We claim that the subproduct $K^{\prime}$ of $G \circ H$ is a clique separator in $G \circ H$. Let $g, g^{\prime}$ be two vertices lying in two different components of $G-K$. Consider the graph $(G \circ H)-(K \circ H)$. Now there is no path between any two vertices $(g, x)$ and $\left(g^{\prime}, y\right)$ in $(G \circ H)-(K \circ H)$, because of the definition of the lexicographic product and since there is no path between $g$ and $g^{\prime}$ in $G-K$. Thus $K \circ H$ is a clique separator in $G \circ H$.

Suppose $G \circ H$ has a clique separator $K^{\prime}$ and suppose for the moment that $H$ is a non-complete graph. For any vertex $g$ of $G$ the $H$ layer ${ }^{g} H$ is not properly contained in $K^{\prime}$. Choose any two vertices $\left(g_{1}, h_{1}\right)$ and $\left(g_{k}, h_{k}\right)$ of $(G \circ H)-K^{\prime}$. If $g_{1} \ldots g_{k}$ 
is a $g_{1}, g_{k}$-path in $G$, then there exists $h_{2}, \ldots, h_{k-1}$ in $H$ so that $\left(g_{1}, h_{1}\right) \ldots\left(g_{k}, h_{k}\right)$ is a $\left(g_{1}, h_{1}\right),\left(g_{k}, h_{k}\right)$-path in $(G \circ H)-K^{\prime}$ - a contradiction with the fact that $K^{\prime}$ is a clique separator of $G \circ H$. Hence $H$ has to be complete.

Now it remains to prove that $G$ has a clique separator. Clearly $(G \circ H)-K^{\prime}$ and $\left\langle p_{G}\left((G \circ H)-K^{\prime}\right)\right\rangle$ are not connected graphs. On the other hand $p_{G}\left(K^{\prime}\right)$ induces a clique in $G$, and since $K=G-p_{G}\left((G \circ H)-K^{\prime}\right) \subseteq\left\langle p_{G}\left(K^{\prime}\right)\right\rangle, K$ is the desired clique separator of $G$.

Note that in the above proof there can exist a clique separator $Y$ in $G \circ H$ such that $p_{G}(Y)$ is not a (clique) separator of $G$. For instance, $P_{3} \circ K_{2}$ has a 3vertex clique separator that properly contains the middle $K_{2}$ layer and so is not minimal. However, $K=G-p_{G}\left((G \circ H)-K^{\prime}\right)$ is a clique separator in $G$ as long as $K^{\prime}$ is a clique separator of $G \circ H$. This phenomena cannot occur if we restrict to the minimal clique separators as the next result shows.

Proposition 3.4. Let $G$ be a connected graph and $H$ a graph. The nontrivial lexicographic product $G \circ H$ has a minimal clique separator $K^{\prime}$ if and only if $K^{\prime}=$ $K \circ H$, where $K$ is a minimal clique separator of $G$ and $H$ is a complete graph.

Proof. Let $K^{\prime}$ be a minimal clique separator of $G \circ H$. By Proposition 3.3, $H$ is complete. It is clear that any clique separator of $G \circ H$ contains at least one $H$ layer. Say that $K^{\prime}$ contains an $H$ layer ${ }^{g} H$. If ${ }^{g} H=K^{\prime}$, then $g$ is a cut vertex in $G$, since $K^{\prime}$ is a clique separator. Hence $K^{\prime}$ is the desired subproduct $\{g\} \circ H$. Now suppose that $K^{\prime} \neq{ }^{g} H$. If $\left(g^{\prime}, h\right) \in K^{\prime}$ and $g \neq g^{\prime}$, then ${ }^{\prime} H \subseteq K^{\prime}$. Indeed, if not, then $\left(g^{\prime}, h^{\prime}\right) \notin K^{\prime}$ for some $h^{\prime} \in V(H)$ and since $(G \circ H)-K^{\prime}$ is not connected, then also $(G \circ H)-\left(K^{\prime}-\left\{\left(g^{\prime}, h\right)\right\}\right)$ is not connected, a contradiction with the minimality of $K^{\prime}$. Thus $K^{\prime}$ is a subproduct $K \circ H$. Furthermore, by Proposition $3.3, K$ is a clique separator of $G$ and if $K$ is not a minimal clique separator of $G$, there exists $K^{*} \subset K$ that is a clique separator of $G$. This yields a contradiction with the minimality of $K^{\prime}=K \circ H$ again, since $K^{*} \circ H$ is a clique separator of $G \circ H$ by Proposition 3.3, which is properly contained in $K^{\prime}$.

Conversely assume that $K$ is a minimal clique separator of $G$ and $H$ is a complete graph. If $K^{\prime}=K \circ H$ is not a minimal clique separator of $G \circ H$, then let $K^{\prime \prime}$ be a clique separator of $G \circ H$ which is contained in $K$. Thus $G-\left(p_{G}((G \circ H)-\right.$ $\left.\left.K^{\prime \prime}\right)\right)$ is a clique separator of $G$ and $G-\left(p_{G}\left((G \circ H)-K^{\prime \prime}\right)\right) \subset K$, a contradiction to the minimality of $K$.

Having exactly described minimal clique separators of the lexicographic product, we can do the same with maximal atoms of that product.

Theorem 3.5. Let $G$ be a connected graph and $H$ a graph. The nontrivial lexicographic product $G \circ H$ has a maximal atom $A^{\prime}$ if and only if either $A^{\prime}=G \circ H$ and $H$ is not complete or $A^{\prime}=A \circ H$, where $A$ is a maximal atom in $G$ and $H$ is complete.

Proof. If $H$ is not complete, then, by Proposition 3.3, it follows that $G \circ H$ has no clique separators, and hence $G \circ H$ is itself an atom (which is also maximal). So 
let $H$ be a complete graph and $A$ a maximal atom in $G$. Again by the Proposition 3.3 , it is clear, that a subgraph of $G \circ H$ of the form $A \circ H$ is a maximal atom in $G \circ H$.

To prove the converse, suppose that $A^{\prime}$ is a maximal atom in $G \circ H$. Again, if $H$ is not complete, $G \circ H$ has no clique separators by Proposition 3.3 and hence $G \circ H$ is a maximal atom. So let $H$ be a complete graph. We show that $\left\langle p_{G}\left(A^{\prime}\right)\right\rangle$ contains no clique separators. If not, let $K$ be a clique separator of $\left\langle p_{G}\left(A^{\prime}\right)\right\rangle$, and consider vertices $g_{1}$ and $g_{2}$ lying in different components of $\left\langle p_{G}\left(A^{\prime}\right)\right\rangle-K$. Clearly $A^{\prime} \cap(K \circ H)$ is a clique in $A^{\prime}$. For any two vertices $\left(g_{1}, h_{1}\right)$ and $\left(g_{2}, h_{2}\right)$ of $A^{\prime}$ there exists no path between them in $A^{\prime}-\left(A^{\prime} \cap(K \circ H)\right)$ - a contradiction with the fact that $A^{\prime}$ is an atom. Thus $\left\langle p_{G}\left(A^{\prime}\right)\right\rangle$ is an atom in $G$ and $\left\langle p_{G}\left(A^{\prime}\right)\right\rangle$ contains no clique separators. By Proposition 3.3 also $\left\langle p_{G}\left(A^{\prime}\right)\right\rangle \circ H$ has no clique separators and $A^{\prime}$ must be equal to $\left\langle p_{G}\left(A^{\prime}\right)\right\rangle \circ H$ (since $A^{\prime}$ is a maximal atom in $G$ ). If $\left\langle p_{G}\left(A^{\prime}\right)\right\rangle$ is not a maximal atom in $G$, there exists an atom, say $U$, containing $\left\langle p_{G}\left(A^{\prime}\right)\right\rangle$. Again we get a larger subgraph $U \circ H$ containing $A^{\prime}$ without clique separators, a contradiction to the maximality of $A^{\prime}$. Hence $A^{\prime}=A \circ H$, where $A$ is a maximal atom in $G$.

\section{THE STRONG PRODUCT}

In [15], ŠPACAPAN recently described minimal separating sets (not necessarily cliques) of $G \otimes H$ as $I$-sets (a subproduct $G \otimes H^{\prime}$ for a proper subgraph $H^{\prime}$ of $H$ or a subproduct $G^{\prime} \otimes H$ for a proper subgraph $G^{\prime}$ of $\left.G\right)$ or $L$-sets $\left(\left(G^{\prime} \otimes H^{\prime}\right)-\left(G^{\prime \prime} \otimes H^{\prime \prime}\right)\right.$ for a proper subgraph $G^{\prime \prime}$ of $G^{\prime}$, which is again a proper subgraph of $G$, and similarly for $H^{\prime \prime}, H^{\prime}$, and $H$ ). In this section we show that a similar result holds for minimal clique separators. Also we can use the previous section for a partial result. Namely $G \circ K_{n} \cong G \otimes K_{n} \cong K_{n} \otimes G$. But first we observe a simple fact about cliques in the strong product.

Lemma 4.6. The nontrivial strong product $G \otimes H$ of connected graphs $G$ and $H$ has clique $\langle K\rangle$ if and only if $p_{G}(K)$ and $p_{H}(K)$ induce cliques in $G$ and $H$, respectively.

Proof. Since $K_{n} \otimes K_{m} \cong K_{n m}$, one direction is trivial. So suppose that $K$ is a clique in $G \otimes H$ and let $g, g^{\prime} \in p_{G}(K)$. If $g=g^{\prime}$, there is nothing to prove. So let $g \neq g^{\prime}$ and let $g=p_{G}((g, h))$ and $g^{\prime}=p_{G}\left(\left(g^{\prime}, h^{\prime}\right)\right)$. Since $(g, h)\left(g^{\prime}, h^{\prime}\right) \in E(G \otimes H)$ and $g \neq g^{\prime}$, we have $g g^{\prime} \in E(G)$ and $\left\langle p_{G}(K)\right\rangle$ is a complete graph. By symmetry, $\left\langle p_{H}(K)\right\rangle$ is also complete.

We continue with a small example of a clique separator that we generalize later. Let $g^{\prime}$ and $h^{\prime}$ be pendant vertices of $G$ and $H$, respectively, with $g g^{\prime}$ and $h h^{\prime}$ being the edges incident with $g^{\prime}$ and $h^{\prime}$, respectively. In the strong product $G \otimes H$, these edges force a $K_{4}$ in $G \otimes H$ and by removing the triple $(g, h)\left(g, h^{\prime}\right)\left(g^{\prime}, h\right)$ from $G \otimes H$, we get an isolated vertex $\left(g^{\prime}, h^{\prime}\right)$. Hence $K_{3}=(g, h)\left(g, h^{\prime}\right)\left(g^{\prime}, h\right)$ is a (minimal) clique separator in $G \otimes H$ if at least one of $G$ and $H$ in not isomorphic to $K_{2}$ and we have the following remark. 
REMARK 4.7. If $G$ and $H$ are noncomplete connected graphs containing pendant vertices then the complete graph $K_{3}$ forms a (minimal) clique separator in the strong product $G \otimes H$.

We can generalize this observation even further. A clique $K$ of a noncomplete graph $G$ is called a pendant clique if $K \nsubseteq N^{G}(G-K)$. In other words, $K$ is a pendant clique of $G$ whenever there exists a vertex $u \in K$ for which $N^{G}(u) \subseteq K$. We call such a vertex $u$ a pendant clique vertex and denote the set of all pendant clique vertices of $K$ with $K^{p}$. In particular, we need to exclude complete graphs from the definition, since they satisfy the definition but have no clique separators. Also note that every pendant clique $K$ with the set of pendant clique vertices $K^{p}$ of a noncomplete graph $G$ contains a clique separator $K^{\prime}=K-K^{p}$.

Now, we move on to describe clique separators in the strong product.

Theorem 4.8. The nontrivial strong product $G \otimes H$ of connected graphs $G$ and $H$ has a minimal clique separator $K$ if and only if either

(i) $K=G \otimes K_{H}$ where $G$ is complete and $H$ has a minimal clique separator $K_{H}$, or

(ii) $K=K_{G} \otimes H$ where $H$ is complete and $G$ has a minimal clique separator $K_{G}$, or

(iii) $K=K_{G} \otimes K_{H}-K_{G}^{p} \otimes K_{H}^{p}$ where $K_{G}$ and $K_{H}$ are pendant cliques in $G$ and $H$, respectively.

Proof. Suppose that $G \otimes H$ has a minimal clique separator $K$. By Lemma 4.6 $\left\langle p_{G}(K)\right\rangle$ and $\left\langle p_{H}(K)\right\rangle$ are complete graphs and at most one equals to $G$ and $H$, respectively. Let first $K_{n} \cong\left\langle p_{H}(K)\right\rangle=H$. Clearly (ii) (and (i) by commutativity of the strong product) follows from Proposition 3.4.

Suppose now that both $G$ and $H$ are not complete graphs. Let $\left(g_{1}, h_{1}\right)$ and $\left(g_{k}, h_{k}\right)$ be vertices of two different components of $(G \otimes H)-K$. By Lemma 4.6 $\left\langle p_{G}(K)\right\rangle=K_{G}$ and $\left\langle p_{H}(K)\right\rangle=K_{H}$ are complete graphs. Hence neither $\left(g_{1}, h_{1}\right)$ nor $\left(g_{k}, h_{k}\right)$ are in $K_{G} \times K_{H}$. If $\left(g_{1}, h_{1}\right) \notin V\left(K_{G} \times K_{H}\right)$, then at least one of the layers ${ }^{g_{1}} H$ or $G^{h_{1}}$, say $G^{h_{1}}$, has an empty intersection with $K$. Moreover, in $G^{h_{1}}$ there exists a vertex $\left(g, h_{1}\right)$ for which also ${ }^{g} H$ has an empty intersection with $K$. Now for every vertex $\left(g^{\prime}, h^{\prime}\right)$ of $A=(V(G) \times V(H))-\left(V\left(K_{G}\right) \times V\left(K_{H}\right)\right)$ at least one layer ${ }^{g^{\prime}} H$ or $G^{h^{\prime}}$ has an empty intersection with $V\left(K_{G} \times K_{H}\right)$, and this layer then has a non-empty intersection with $G^{h_{1}}$ or ${ }^{g} H$. Thus vertices of $A$ induce a connected graph and $\left(g_{k}, h_{k}\right)$ must be from $V\left(K_{G}\right) \times V\left(K_{H}\right)$ which is a clique. Moreover, $g_{k}$ and $h_{k}$ must be pendant clique vertices of $K_{G}$ and $K_{H}$, respectively, otherwise they would be adjacent to a vertex of $A$ which is impossible. Thus $V(K) \subset V\left(K_{G}\right) \times V\left(K_{H}\right)$, where $K_{G}$ and $K_{H}$ are pendant cliques of $G$ and $H$, respectively. Clearly every vertex $\left(g^{\prime \prime}, h^{\prime \prime}\right) \in\left(V\left(K_{G}\right) \times V\left(K_{H}\right)\right)-\left(V\left(K_{G}^{p}\right) \times V\left(K_{H}^{p}\right)\right)$ must be in $K$, otherwise there would be a $\left(g_{1}, h_{1}\right),\left(g_{k}, h_{k}\right)$-path in $(G \otimes H)-K$. Hence $K \supseteq\left(K_{G} \otimes K_{H}\right)-\left(K_{G}^{p} \otimes K_{H}^{p}\right)$. On the other hand, $\left(K_{G} \otimes K_{H}\right)-\left(K_{G}^{p} \otimes K_{H}^{p}\right)$ is a clique separator since it separates $K_{G}^{p} \otimes K_{H}^{p}$ from $A$. Thus $K=\left(K_{G} \otimes K_{H}\right)-$ $\left(K_{G}^{p} \otimes K_{H}^{p}\right)$ and (iii) follows. 
Conversely it is clear for (i) that $K=G \otimes K_{H}$ is a clique separator when $G$ is a complete graph and $K_{H}$ a clique separator of $H$. Moreover, $K$ is minimal, since $K_{H}$ is minimal. Similarly for (ii). For (iii) note that $K=\left(K_{G} \otimes K_{H}\right)-\left(K_{G}^{p} \otimes K_{H}^{p}\right)$ is a clique separator that separates $K_{G}^{p} \otimes K_{H}^{p}$ and $(V(G) \times V(H))-\left(V\left(K_{G}\right) \times V\left(K_{H}\right)\right)$ for pendant cliques $K_{G}$ and $K_{H}$ of $G$ and $H$, respectively. The minimality follows immediately since the product of all pendant vertices is excluded.

Note that the strong product of typical graphs has no (minimal) clique separators. This holds if both factors are complete graphs, one is complete and the other has no clique separator, or at least one has no pendant clique. Using Theorem 4.8, we can also describe the structure of all clique separators of the strong product. If $G \otimes H$ has a maximal atom $A$, one factor, say $G$, is a complete graph and the other has a minimal clique separator $K_{H}$, and $K=\left(G \otimes K_{H}\right) \cup A$ induces a connected graph, then it is clear that $K$ is a clique separator if and only if either $\left\langle p_{H}(K)\right\rangle$ is not a pendant clique of $H$ or $\left\langle p_{H}(K)\right\rangle$ is a pendant clique and there exists $(g, h) \notin K$ for some $h \in\left\langle p_{H}(K)\right\rangle^{p}$. Indeed, if $\left\langle p_{H}(K)\right\rangle$ is not a pendant clique, the part $G \otimes K_{H}$ insures that $K$ is a separator and $p_{H}(K)$ insures that $K$ is a clique. Otherwise, if $p_{H}(K)$ is a pendant clique, the fact that $(g, h) \notin K$ for some $h \in\left\langle p_{H}(K)\right\rangle^{p}$ guarantees that $(G \otimes H)-K$ is not connected.

If $G$ and $H$ are not complete, note that we need a pendant clique in each factor to assure the clique separator in the strong product. Thus $K$ is a clique separator if and only if the following condition holds

$$
\left(K_{G} \otimes K_{H}\right)-\left(K_{G}^{p} \otimes K_{H}^{p}\right) \subseteq K \subset K_{G} \otimes K_{H},
$$

where $K_{G}$ and $K_{H}$ are some pendant cliques in $G$ and $H$, respectively. Clearly $K$ is a clique and the proper subset guarantees that $K$ also separates the product.

Next we describe all maximal atoms of the strong product $G \otimes H$.

Theorem 4.9. The nontrivial strong product $G \otimes H$ of connected graphs $G$ and $H$ has a maximal atom $A$ if and only if either

(i) $A=G \otimes A_{H}$ where $G$ is complete and $H$ has a proper maximal atom $A_{H}$, or

(ii) $A=A_{G} \otimes H$ where $H$ is complete and $G$ has a proper maximal atom $A_{G}$, or

(iii) $A=K_{G} \otimes K_{H}$ where $K_{G}$ and $K_{H}$ are pendant cliques in $G$ and $H$, respectively, or

(iv) $A=(G \otimes H)-\left(P_{G} \times P_{H}\right)$ where $P_{G}$ and $P_{H}$ are sets of all pendant clique vertices of $G$ and $H$, respectively, or

(v) $A=G \otimes H$.

Proof. Note that conditions (i), (ii), (iii), (iv), and (v) have pairwise empty intersections. For instance in (i) and (ii) the proper maximal atom is needed which differentiates (i) and (ii) from (v). First, let $A$ be a maximal atom in $G \otimes H$. Thus there is no clique separator in $A$. By Theorem 4.8 the following disjoint possibilities for clique separators may occur: either one factor is complete and the other contains 
a proper minimal clique separator, or $G$ and $H$ have both some pendant cliques, or there is no proper clique separator in $G \otimes H$. The last case clearly yields (v) and the first case yields (i) or (ii), respectively, since the separation by minimal clique separators implies proper atoms (which are contained in proper maximal atoms). The middle case implies that $G$ and $H$ are not complete. Let $(g, h) \in A$ for pendant clique vertices $g$ of $G$ and $h$ of $H$. If $g \in K_{G}$ and $h \in K_{H}$ for pendant cliques $K_{G}$ and $K_{H}$, then $K_{G} \otimes K_{H} \subseteq A$ by (iii) of Theorem 4.8 and furthermore, $K_{G} \otimes K_{H}=A$ by the same reason. Hence (iii) follows. If no such vertex exists in $A$, we only need to see that $A=(G \otimes H)-\left(P_{G} \times P_{H}\right)$ is an atom, since every additional vertex would yield the previous case (iii). If $A$ contains a clique separator $K$, then $K$ is also a clique separator of $G \otimes H$, since every pendant clique vertex belongs to a unique maximal pendant clique. But this is a contradiction with Theorem 4.8.

Suppose now that either (i), (ii), (iii), (iv), or (v) holds. If $G$ (or $H$ ) is complete we can use the argument in the proof of Theorem 3.5 to see that $A=$ $K_{n} \otimes A_{H}$ (or $A=A_{G} \otimes K_{n}$ ) is a maximal atom for a proper maximal atom $A_{G}$ (or $A_{H}$ ) of $G$ (or $H$ ).

If (iii) is fulfilled, note that $G$ and $H$ are not complete, $A$ is a clique and that by (iii) of Theorem $4.8 K=A-\left(K_{G}^{p} \otimes K_{H}^{p}\right)$ is a clique separator of $G \otimes H$, where $K_{G}$ and $K_{H}$ are pendant cliques of $G$ and $H$, respectively. Hence $A$ has no clique separator and is maximal, since otherwise $K$ would be a clique separator in $A \cup\{(g, h)\}$, where $(g, h) \notin A$ is an arbitrary vertex adjacent to a vertex of $A$.

Let now $A=(G \otimes H)-\left(P_{G} \times P_{H}\right)$ for the sets of all pendant clique vertices $P_{G}$ and $P_{H}$ of $G$ and $H$, respectively. Clearly $A$ is maximal in the sense that $A \cup\{(g, h)\}$, $(g, h) \notin A$, contains a clique separator $K=\left(K_{G} \otimes K_{H}\right)-\left(K_{G}^{p} \otimes K_{H}^{p}\right)$ by (iii) of Theorem 4.8, where $K_{G}$ and $K_{H}$ are pendant cliques of $G$ and $H$, respectively, and $g \in K_{G}^{p}$ and $h \in K_{H}^{p}$. If $A$ is not an atom, then there exists a clique $K$ in $A$, which separate $A$. Again this yields a contradiction with Theorem 4.8, since $K$ would then be a clique separator of $G \otimes H$ by unique membership in a maximal pendant clique of each pendant clique vertex.

Finally, let $A=G \otimes H$. Note that all cases from Theorem 4.8 have been covered by (i), (ii), (iii), and (iv) and thus it remains that $G \otimes H$ contains no clique separator. Hence $A$ is the maximal atom.

\section{THE DIRECT PRODUCT}

The minimal separation by vertices of the direct product is a tough problem as observed by BRES̆AR and S̆PACAPAN in [5]. This is not the case if we restrict ourselves to the clique separators, since no direct product has a clique separator with three or more vertices, as we will see in Theorem 5.12.

For the direct product every clique $K_{n}$ of $G \times H$ clearly implies cliques of the same order in the factors $G$ and $H$, since every edge of $G \times H$ is projected to an edge in every factor and two vertices of the same layer are nonadjacent. On the other hand the direct product is not always a connected graph even if both factors are. Indeed, $G \times H$ is a connected graph if and only if at least one of $G$ and $H$ is non bipartite. Moreover, if both $G$ and $H$ are bipartite, $G \times H$ has exactly 
two components - see [17] and also [11] — where vertices $(g, h)$ and $\left(g, h^{\prime}\right)$ with $h h^{\prime} \in E(H)$ are in different components, as are $(g, h)$ and $\left(g^{\prime}, h\right)$ with $g g^{\prime} \in E(G)$.

Lemma 5.10. Let $G$ and $H$ be connected graphs, each on at least three vertices. If $(g, h)$ is a cut vertex of $G \times H$, then $g$ and $h$ are cut vertices in $G$ and $H$, respectively.

Proof. Suppose that $g$ is not a cut vertex of $G$ but $(g, h)$ is a cut vertex of $G \times H$. Let $\left(g_{0}, h_{0}\right)$ and $\left(g_{k}, h_{k}\right)$ be in different components of $(G \times H)-\{(g, h)\}$ but there exists a path $P=\left(g_{0}, h_{0}\right) \ldots\left(g_{k}, h_{k}\right)$ in $G \times H$. Moreover, every $\left(g_{0}, h_{0}\right),\left(g_{k}, h_{k}\right)$ path in $G \times H$ contains $(g, h)$. Note that $p_{G}(P)$ induces a $g_{1}, g_{k}$-walk in $G$. Since $g$ is not a cut vertex, there exists a $g_{0}, g_{k}$-path $Q=g_{0} g_{1}^{\prime} \ldots g_{i}^{\prime} g_{k}$ in $G$ for which $g \notin Q$. If $Q$ and $P$ have the same parity, we have, for $i+1=|Q| \geq|P|=k$, a $\left(g_{0}, h_{0}\right),\left(g_{k}, h_{k}\right)$-path

$$
\left(g_{0}, h_{0}\right)\left(g_{1}^{\prime}, h_{1}\right) \ldots\left(g_{k}^{\prime}, h_{k}\right)\left(g_{k+1}^{\prime}, h_{k-1}\right)\left(g_{k+2}^{\prime}, h_{k}\right) \ldots\left(g_{i}^{\prime}, h_{k-1}\right)\left(g_{k}, h_{k}\right)
$$

in $G \times H$ that does not contain $(g, h)$ which is a contradiction. Similar for $i+1<k$ the path

$$
\left(g_{0}, h_{0}\right)\left(g_{1}^{\prime}, h_{1}\right) \ldots\left(g_{i}^{\prime}, h_{i}\right)\left(g_{k}, h_{i+1}\right)\left(g_{i}^{\prime}, h_{i+2}\right)\left(g_{k}, h_{i+3}\right) \ldots\left(g_{i}^{\prime}, h_{k-1}\right)\left(g_{k}, h_{k}\right)
$$

yields a contradiction again.

Hence $Q$ and $P$ must have different parity. In particular $Q$ and $p_{G}(P)$ form an odd closed walk and $G$ is not a bipartite graph. But then in $\left(Q \cup p_{G}(P)\right) \times\left\{h_{0} h_{1}\right\}$ there exist at least two different $\left(g_{0}, h_{0}\right),\left(g_{0}, h_{1}\right)$-paths $P_{1}$ and $P_{2}$. The first starts in the first coordinate along $Q$ and the second along $p_{G}(P)$ and exchanging the second coordinate. Moreover $(g, h)$ is on at most one of them (if $h=h_{0}$ or $h=h_{1}$ ). Suppose that $(g, h)$ is not on $P_{1}$. The walk $p_{H}(P)$ is a walk in $H$ between $h_{0}$ and $h_{k}$ and contains a walk between $h_{1}$ and $h_{k}$ that is of the same parity as $Q$. Thus there exists a path $P^{\prime}$ between $\left(g_{0}, h_{1}\right)$ and $\left(g_{k}, h_{k}\right)$ in $G \times H$ with $(g, h) \notin P^{\prime}$. But then $P_{1} \cup P^{\prime}$ induces a $\left(g_{0}, h_{0}\right),\left(g_{k}, h_{k}\right)$-walk in $(G \times H)-\{(g, h)\}$, a final contradiction.

Lemma 5.11. No edge is a (clique) separator of $C_{n} \times P_{3}$ for $n \geq 3$.

Proof. Let $P_{3}=h h^{\prime} h^{\prime \prime}$ and $C_{n}=g_{1} \ldots g_{n}$. First, note by symmetry that we only have two types of edges $\left(g_{i}, h\right)\left(g_{i+1}, h^{\prime}\right)$ or $\left(g_{i}, h^{\prime}\right)\left(g_{i+1}, h\right)$. If $n$ is odd, $C_{n} \times P_{2}$ is a cycle on $2 n$ vertices. Clearly $\left(C_{n} \times h h^{\prime}\right)-e$ and $\left(C_{n} \times h^{\prime} h^{\prime \prime}\right)-e$ are paths on at least $2 n-2$ vertices. For $n \geq 3, n-1$ vertices are common to both $\left(C_{n} \times h h^{\prime}\right)-e$ and $\left(C_{n} \times h^{\prime} h^{\prime \prime}\right)-e$. Hence $\left(C_{n} \times P_{3}\right)-e$ is connected. If $n$, for $n \geq 4$, is an even number, then $C_{n} \times P_{3}$ and $C_{n} \times P_{2}$ have two components. Each component of $C_{n} \times P_{2}$ is a cycle and in $\left(C_{n} \times P_{2}\right)-e$ these cycles remain the same or convert to paths for $P_{2}=h h^{\prime}$ or $P_{2}=h^{\prime} h^{\prime \prime}$. These components have at least one vertex in $C_{n}^{h^{\prime}}$ layer in common, since $n \geq 4$ and $\left(C_{n} \times P_{3}\right)-e$ has two components.

Before we state the result on the direct product we add the notation $G / e$, which is a graph obtained from graph $G$ by contracting a fixed edge $e$. Also recall that for a cut vertex $g \in G$ and a component $C$ of $G-\{g\}$, we denote $C^{g}=$ $\langle C \cup\{g\}\rangle$. 
Theorem 5.12. Let $G$ and $H$ be connected graphs, each on at least three vertices. The direct product $G \times H$ has a minimal clique separator $K$ if and only if either

(i) $K=\{(g, h)\}$ where $g$ and $h$ are cut vertices of $G$ and $H$, respectively, that are adjacent to a pendant vertex, or

(ii) $K=\{(g, h)\}$ where $g$ is a cut vertex of $G$ not adjacent to a pendant vertex, $G-\{g\}$ has at least one bipartite component $C^{g}$, and $h$ is a central vertex of $K_{1, n} \cong H$, or

(iii) $K=\left\{(g, h),\left(g^{\prime}, h^{\prime}\right)\right\}$ where $g, g^{\prime}$ and $h, h^{\prime}$ are adjacent cut vertices of $G$ and $H$, respectively, $N_{G}(g)-\left\{g^{\prime}\right\}$ and $N_{H}\left(h^{\prime}\right)-\{h\}$ consist of pendant vertices, and $g^{\prime}$ and $h$ are not adjacent to any pendant vertex, or

(iv) $K=\left\{(g, h),\left(g^{\prime}, h^{\prime}\right)\right\}$ where $g$ and $g^{\prime}$ are adjacent cut vertices of $G$ not adjacent to any pendant vertex, $G-\{g\}$ has at least one bipartite component $C^{g}$, and $h h^{\prime} \in V\left(H / h h^{\prime}\right)$ is a central vertex of $K_{1, n} \cong H / h h^{\prime}$ with $\operatorname{deg}_{H} h>1$ and $\operatorname{deg}_{H} h^{\prime}>1$.

Proof. First, note that the conditions (i), (ii), (iii), and (iv) have pairwise empty intersections, because of the requirements, whether vertices have a pendant vertex in their neighborhood or not. Suppose that either (i), (ii), (iii), or (iv) is fulfilled. If $K=\{(g, h)\}$ for cut vertices $g$ and $h$ of $G$ and $H$, respectively, that are adjacent to a pendant vertex $g^{\prime}$ and $h^{\prime}$, respectively, then $\left(g^{\prime}, h^{\prime}\right)$ is a pendant vertex of $G \times H$ adjacent to $(g, h)$. Clearly $(G \times H)-K$ has more components than $G \times H$, and $K$ is a minimal clique separator.

Let $h$ be a central vertex of $H \cong K_{1, n}, g$ a cut vertex of $G$ with no pendant vertex in its neighborhood, and $C^{g}$ a bipartite component of $G-\{g\}$. Denote with $h_{1}, \ldots, h_{n}$ all pendant vertices of $H$ and let $C^{\prime}$ be a component of $G-\{g\}$ different from $C^{g}$. Furthermore, let $A_{2 i-1}=\left\{\left(g^{\prime}, h_{j}\right): g^{\prime} \in S_{2 i-1}^{C^{g}}(g)\right\}$ and $A_{2 i}=\left\{\left(g^{\prime}, h\right)\right.$ : $\left.g^{\prime} \in S_{2 i}^{C^{g}}(g)\right\}$ for $i \in \mathbb{N}$. Note that $p_{X}\left(A_{2 i-1}\right)$ and $p_{X}\left(A_{2 i}\right)$, for $X \in\{G, H\}$, induce the partitions of $V(H)$ and $V\left(C^{g}\right)$. Thus $A=\left\langle\cup_{i \in \mathbb{N}} A_{i}\right\rangle$ form a component separated by $(g, h)$ from the graph $B=(G \times H)-(A \cup\{(g, h)\})$. Indeed, the projection of any edge between a vertex from $A$ and a vertex from $B$ implies, that $g$ is not a cut vertex of $G$ or an edge between two vertices of $p_{X}\left(A_{2 i-1}\right)$ or $p_{X}\left(A_{2 i}\right)$, for $X \in\{G, H\}$, which is impossible. Thus $K=\{(g, h)\}$ is a (minimal) clique separator.

Let $K=\left\{(g, h),\left(g^{\prime}, h^{\prime}\right)\right\}$ where $g, g^{\prime}$ and $h, h^{\prime}$ are adjacent cut vertices of $G$ and $H$ respectively. If $N_{G}(g)-\left\{g^{\prime}\right\}=\left\{g_{i}: i \in\left\{1, \ldots, i_{g}\right\}\right\}$ and $N_{H}\left(h^{\prime}\right)-\{h\}=$ $\left\{h_{i}: i \in\left\{1, \ldots, i_{h}\right\}\right\}$ consist of pendant vertices, then $A=\left\{\left(g, h_{i}\right),\left(g_{j}, h^{\prime}\right)\right.$ : $\left.j \in\left\{1, \ldots, i_{g}\right\}, i \in\left\{1, \ldots, i_{h}\right\}\right\}$ forms a component not adjacent to any vertex of $(G \times H)-(A \cup K)$. Thus $K$ is a clique separator. Moreover, $\{(g, h)\}$ and $\left\{\left(g^{\prime}, h^{\prime}\right)\right\}$ are not clique separators since $g^{\prime}$ and $h$ are cut vertices not adjacent to any pendant vertex.

Let $K=\left\{(g, h),\left(g^{\prime}, h^{\prime}\right)\right\}$ for adjacent cut vertices $g$ and $g^{\prime}$ of $G$. Furthermore, let $C^{g}$ be a bipartite component of $G-\{g\}$ and $h h^{\prime} \in V\left(H / h h^{\prime}\right)$ a central vertex of $K_{1, n} \cong H / h h^{\prime}$. In particular $h h^{\prime} \in E(H)$ and $\langle K\rangle$ is an edge. Clearly $N^{H}\left(h^{\prime}\right)-$ $\{h\}$ and $N^{H}(h)-\left\{h^{\prime}\right\}$ contain only pendant vertices of $H$. We claim that for $A_{2 i+1}=\left\{\left(g_{j}, h_{k}\right): g_{j} \in S_{2 i+1}^{C^{g}}(g), h_{k} \in N^{H}(h)\right\}$ and $A_{2 i}=\left\{\left(g_{j}, h_{k}\right): g_{j} \in\right.$ $\left.S_{2 i}^{C^{g}}(g), h_{k} \in N^{H}\left(h^{\prime}\right)\right\}$ for $i \in \mathbb{N}_{0}$, the set $A=\left(\cup_{i \in \mathbb{N}_{0}} A_{i}\right)-\{(g, h)\}$ is separated 
from $(G \times H)-(A \cup K)$ by $K$. First, note that all vertices of $A$, that have neighbors outside of $A$, are $\left(g, h_{k}\right)$ for $h_{k} \in N^{H}\left(h^{\prime}\right)-\{h\}$ or $\left(g_{j}, h_{k}\right)$ for $g_{j} \in N^{C_{g}}(g)$ and $h_{k} \in N^{H}(h)$. First ones are adjacent only to $\left(g^{\prime}, h^{\prime}\right)$, since $g$ and $g^{\prime}$ are cut vertices and $N^{H}\left(h^{\prime}\right)-\{h\}$ contains only pendant vertices. Similarly the second ones are adjacent only to $(g, h)$, since $g$ is a cut vertex and $N^{H}(h)-\left\{h^{\prime}\right\}$ contains only pendant vertices. Hence $K$ is a clique separator and moreover it is a minimal since $g$ and $g^{\prime}$ have no pendant vertices for neighbors.

Conversely, let $K$ be a minimal clique separator with $k=|K| \geq 3$. We gain the contradiction in which we show that $(G \times H)-K$ is a connected graph. Clearly $p_{G}(K)$ and $p_{H}(K)$ are cliques $K_{k}^{G}$ and $K_{k}^{H}$, respectively, and $G$ and $H$ are non bipartite. If $\left(g_{1}, h_{1}\right),\left(g_{2}, h_{2}\right),\left(g_{3}, h_{3}\right) \in K$, then $g_{1}, g_{2}$, and $g_{3}\left(h_{1}, h_{2}\right.$, and $\left.h_{3}\right)$ are different vertices of $G(H)$. Let $(i, j, k)$ be any permutation of $\{1,2,3\}$. Note that $\left(g_{i}, h_{j}\right)$ and $\left(g_{i}, h_{k}\right)$ have a common neighbor $\left(g_{j}, h_{i}\right)$ in $(G \times H)-K$. Similarly $\left(g_{i}, h_{j}\right)$ and $\left(g_{k}, h_{j}\right)$ have a common neighbor $\left(g_{j}, h_{i}\right)$ in $(G \times H)-K$ and in particular $\left(\left\langle p_{G}(K)\right\rangle \times\left\langle p_{H}(K)\right\rangle\right)-K$ is connected. Thus we have a contradiction if $G=K_{k}^{G}$ and $H=K_{k}^{H}$. So suppose that at least one, say $G$, is different than $K_{k}^{G}$. If $g \notin K_{k}^{G}$ has a neighbor in $K_{k}^{G}$, say $g_{1}$, then $\left(g, h_{i}\right)$, for $h_{i} \in K_{k}^{H}$ has at least one neighbor in $\left(\left\langle p_{G}(K)\right\rangle \times\left\langle p_{H}(K)\right\rangle\right)-K$. If $H$ is also different than $K_{k}^{H}$, then there exists $h \notin K_{k}^{H}$ adjacent to a vertex in $K_{k}^{H}$, say $h_{1}$. Since $k \geq 3, \operatorname{deg}_{G \times H}(g, h)>1$ (otherwise $\left\{\left(g_{1}, h_{1}\right)\right\}$ is a clique separator contrary to the minimality of $\left.K\right)$. If there is another neighbor of $(g, h)$ in $\left\langle p_{G}(K)\right\rangle \times\left\langle p_{H}(K)\right\rangle$, we are done. Otherwise there is a neighbor $\left(g^{\prime}, h^{\prime}\right)$, either in $K_{k}^{G} \times H$ or in $G \times K_{k}^{H}$. In both cases we have the same situation as before. Furthermore, note that, since $G$ and $H$ are connected, there exists a path from every vertex $\left(g^{*}, h^{*}\right) \in(G \times H)-K$ to either $(g, h)$ or to $\left(g_{1}, h\right)$ in $G \times H$ and by above also in $(G \times H)-K$. Hence $(G \times H)-K$ is connected which is impossible and $|K| \leq 2$.

First, let $|K|=1$ and let $K=\{(g, h)\}$. Clearly $(g, h)$ is a cut vertex and by Lemma 5.10, $g$ and $h$ are cut vertices of $G$ and $H$, respectively. If for both $g$ and $h$ there exists a pendant vertex in their neighborhood, (i) follows. So suppose that for one of them, say $g$, every component $C_{i}$ of $G-\{g\}$ has at least two vertices. If $H$ or all $C_{i}^{g}$ are not bipartite, then $C_{i}^{g} \times H$ is a connected graph and hence $(G \times H)-K$ is connected contrary to the assumption. Thus $H$ is bipartite as well as at least one component, say $C_{1}^{g}$. Let $C^{\prime}$ and $C^{\prime \prime}$ be two components of $C_{1}^{g} \times H$ and let $N^{H}(h)=\left\{h_{i}: i=1, \ldots, n\right\}$. Let, furthermore, $g_{1} \in C_{1}^{g}$ be a neighbor of $g$. If there exists a vertex $h^{\prime} \in S_{2}^{H}(h)$, we assume that $h_{1}$ is a common neighbor of $h$ and $h^{\prime}$. We choose $C^{\prime}$ so that $\left(g_{1}, h_{1}\right) \in C^{\prime}$ and then $\left(g_{1}, h\right) \in C^{\prime \prime}$. But then for any vertex $g^{\prime} \in N^{G-C_{1}^{g}}(g)$ the path $\left(g^{\prime}, h_{1}\right)\left(g, h^{\prime}\right)\left(g_{1}, h_{1}\right)$ is a path from a vertex from $C^{\prime}$ to a vertex from $(G \times H)-\left(\left(C_{1}^{g} \times H\right) \cup\{(g, h)\}\right)$. Since there also exists a path $\left(g_{1}, h\right)\left(g, h_{1}\right)\left(g^{\prime}, h\right)$ from $C^{\prime \prime}$ to $(G \times H)-\left(C_{1}^{g} \times H \cup\{(g, h)\}\right)$, this yields that $(G \times H)-K$ has the same number of components as $G \times H$, which is impossible, since $K$ is a clique separator. (We will call this argument distance 2 argument, since $d_{H}\left(h, h^{\prime}\right)=2$.) Thus all vertices of $H$ are in $N^{H}[h]$ and since $H$ is bipartite we have $H \cong K_{1, n}$. Hence (ii) holds.

Finally let $|K|=2$ and let $K=\left\{(g, h),\left(g^{\prime}, h^{\prime}\right)\right\}$. By Lemma 5.11, each of 
$g, g^{\prime}, h$, and $h^{\prime}$ must be either a cut vertex or a pendant vertex. Moreover, neither $g$ and $g^{\prime}$ nor $h$ and $h^{\prime}$ are pendant vertices since $G$ and $H$ contains at least three vertices. If $N^{G}(g)-\left\{g^{\prime}\right\}$ is non-empty and contains only pendant vertices, then $g$ is a cut vertex in $G$ and $h$ is not adjacent to any pendant vertex, otherwise we have (i) and a contradiction with minimality of $K$. If $N^{H}\left(h^{\prime}\right)-\{h\}$ contains no pendant vertex, then it contains a triangle or there exists a vertex $h_{1}$ with $d_{H}\left(h^{\prime}, h_{1}\right)=2$. The first case is not possible by Lemma 5.11 and the second is not possible by the distance 2 argument. Thus $N^{H}\left(h^{\prime}\right)-\{h\}$ consist only of pendant vertices, $h^{\prime}$ is a cut vertex and $g^{\prime}$ has no pendant neighbor by the same reason as above. In particular $h$ and $g^{\prime}$ are not pendant vertices and are thus cut vertices. But then (iii) holds.

Otherwise, let $g$ be adjacent to a non pendant vertex different than $g^{\prime}$ and is thus a cut vertex by Lemma 5.11. Since $H$ is connected and has at least three vertices, there exists a vertex $h^{*}$ adjacent to either $h$ or $h^{\prime}$. Say first $h h^{*} \in E(H)$ and thus $\operatorname{deg}_{H} h>1$. If $h^{*}$ is not a pendant vertex, we have a contradiction with distance 2 argument (if there exists a neighbor $h_{2}$ of $h^{*}$ with $d_{H}\left(h, h_{2}\right)=2$ ) or a contradiction that $K$ is a (clique) separator by Lemma 5.11 (if $h^{*}$ and $h$ are in common triangle). Hence $N^{H}(h)-\left\{h^{\prime}\right\}$ contains only pendant vertices. Furthermore, $g$ can not be adjacent to any pendant vertex, since we would have (i) and a contradiction with minimality of $K$ again. In particular $g^{\prime}$ is a cut vertex.

Let $C_{i}$, for $i=1, \ldots, k$ be components of $G-\{g\}$. If every $C_{i}^{g}$ is not bipartite, then $C_{i}^{g} \times H$ is a connected graph and hence $(G \times H)-K$ is connected contrary to the assumption. Thus at least one component, say $C_{1}^{g}$, is bipartite. If $\operatorname{deg}_{H} h^{\prime}=1$ we have (ii) and a contradiction with minimality of $K$ again. So $\operatorname{deg}_{H} h^{\prime}>1$. If there is a non pendant vertex in $N^{H}\left(h^{\prime}\right)-\{h\}$ we have a contradiction by distance 2 argument again. Hence also $g^{\prime}$ is not adjacent to any pendant vertex, otherwise we have (i) and a contradiction with minimality of $K$. Thus (iv) follows and the proof is completed.

From the proof of the above theorem it is easy to describe all clique separators. Namely $K$ is a clique separator of $G \times H$ if and only if either $K$ is minimal clique separator or $K$ is an edge containing a cut vertex (which is a minimal clique separator). This is obvious since there are no clique separators on 3 or more vertices. On the other hand the minimal clique separator $K=\{(g, h)\}$ can separate differently as $K^{\prime}=\left\{(g, h),\left(g^{\prime}, h^{\prime}\right)\right\}$. For this, we observe $P_{4} \times P_{4}$ where $g, g^{\prime}, h$, and $h^{\prime}$ are vertices of degree 2 . Graph $\left(P_{4} \times P_{4}\right)-K$ has 3 components, but $\left(P_{4} \times P_{4}\right)-K^{\prime}$ has 5 components. Note that this happens only when (iii) or (iv) are not fulfilled, since $g^{\prime}$ and $h$ are adjacent to some pendant vertices. This must be considered to describe maximal atoms.

There are too many possibilities to describe maximal atoms of the direct product in the similar form as in Theorem 4.9 for the strong product and we proceed step by step. First we consider all direct products without clique separators, i.e. $G \times H$ is a maximal atom. This is easy by considering the opposite of Theorem 5.12. Note that the statement (i) of the following theorem covers (i) and (iii) of Theorem 5.12. 
Theorem 5.13. Let $G$ and $H$ be connected graphs, each on at least three vertices, where at most one of them is bipartite. Graph $G \times H$ is a maximal atom if and only if either

(i) $G$ and $H$ have no pendant vertices, or

(ii) $H \cong K_{1, n}$ and $G$ has no pendant vertices and for every cut vertex $g \in$ $V(G)$ every component $C^{g}$ of $G-\{g\}$ is not bipartite, or

(iii) $h h^{\prime} \in V\left(H / h h^{\prime}\right)$ is a central vertex of $K_{1, n} \cong H / h h^{\prime}$ with $\operatorname{deg}_{H} h>1$ and $\operatorname{deg}_{H} h^{\prime}>1, G$ has no pendant vertices and for every cut vertex $g \in V(G)$ every component $C^{g}$ of $G-\{g\}$ is not bipartite.

Proposition 5.14. An edge $e$ is a maximal atom of $G \times H$ if and only if it is a pendant edge.

Proof. Clearly a pendant edge is a maximal atom. Contrary, if $e=(g, h)\left(g^{\prime}, h^{\prime}\right)$ is a non pendant edge of $G \times H$, then $g$ has a neighbor $g^{\prime \prime}$ in $G$ other than $g^{\prime}$ as well as $h^{\prime}$ has a neighbor $h^{\prime \prime}$ in $H$ other than $h$. The 4-cycle $(g, h)\left(g^{\prime}, h^{\prime}\right)\left(g, h^{\prime \prime}\right)\left(g^{\prime \prime}, h^{\prime}\right)$ does not contain any clique separator and $e$ is not a maximal atom.

An obstacle in describing maximal atom is that the direct product is not connected when both factors are bipartite. But atoms are connected subgraphs. Hence we cannot have a result of the type (maximal) atom is a subproduct of two (maximal) atoms. We only give some directions in the end of this section, that lead to other maximal atoms. Following (iii) of Theorem 5.12 let $g$ and $h^{\prime}$ be cut vertices, where all but possible one, say $g^{\prime}$ and $h$, respectively, neighbors are pendant vertices. The set

$$
\left(\left(N^{G}(g)-\left\{g^{\prime}\right\}\right) \times\left\{h^{\prime}\right\}\right) \cup\left(\{g\} \times\left(N^{H}\left(h^{\prime}\right)-\{h\}\right)\right) \cup\left\{(g, h),\left(g^{\prime}, h^{\prime}\right)\right\}
$$

forms a maximal atom. Moreover, in that case

$$
(G \times H)-\left\{(g, h),\left(g^{\prime}, h\right),\left(g, h^{\prime}\right):(g, h) \in P\right\}
$$

is also a maximal atom for the set $P$ of all pendant vertices of $G \times H$, whenever at least one of $G$ or $H$ is non bipartite and the other is not $K_{1, n}$ or $K_{1, n}$ can be obtained by contracting an edge. Note that $(g, h)$ must be excluded by $(i)$ and the edge $\left(g^{\prime}, h\right)\left(g, h^{\prime}\right)$ by (iii) of Theorem 5.12. The remaining part is not hard but tedious and have many cases since we have to observe only components of the product and take into consideration whether one factor is isomorphic to $K_{1, n}$ or $K_{1, n}$ can be obtained by contracting an edge and is omitted.

\section{ALGORITHM FOR DECOMPOSITION BY MINIMAL CLIQUE SEPARATORS OF LEXICOGRAPHIC PRODUCT AND STRONG PRODUCT}

The decomposition algorithm, that we call BPS algorithm for short, presented in [4] will decompose the graph into maximal atoms and minimal clique separators. 
The algorithm can be described as follows. Decompose a graph with minimal clique separators. The algorithm repeats the decomposition step given below on each of the components until we have no separators.

\section{Algorithm 5.15. Decomposition algorithm}

1. Choose one minimal clique separator in $G$ if such a separator exists.

2. Decompose $G$ into set of subgraphs $\left\{\left\langle C_{i} \cup N^{G}\left(C_{i}\right)\right\rangle: C_{i} \in C(S)\right\}$.

Here $C(S)$ denotes the set of components of $G(V-S)$. The algorithm has complexity $O(m n)$ and it finds all minimal clique separators of $G$. We use this algorithm to find minimal clique separators of $G \circ H$ which is done by the following Algorithm.

Algorithm 5.16. Decomposition by minimal clique separators of lexicographic product

Input: Graphs $G, H$, and $G \circ H$.

Step 1. Check whether $H$ is complete or not. If not then $G \circ H$ has no clique separator by Proposition 3.3.

else

Step 2. Decompose $G$ into maximal atoms by BPS algorithm

Step 3. For each minimal separator $K$ found in Step 2, find $K \circ H$ by restricting the vertices of $H$.

Stop.

Correctness of the algorithm follows from Proposition 3.4. If $G$ has $n_{1}$ vertices and $m_{1}$ edges and $H$ has $n_{2}$ vertices and $m_{2}=n_{2}\left(n_{2}-1\right) / 2$ edges, the complexity of finding the minimal clique separators directly by BPS decomposition algorithm is $O\left(n_{1} n_{2}\left(n_{1} m_{2}+m_{1} n_{2}^{2}\right)\right)$. On the other hand, we need to use the BPS algorithm only for $G$ which gives the complexity $O\left(n_{1} m_{1}\right)$ and is much better.

Now, we describe the decomposition algorithm of strong product.

\section{Algorithm 5.17. Decomposition by minimal clique separators in strong} product

Input: Graphs $G, H$, and $G \otimes H$.

Step 1. If $G$ or $H$ is a complete graph, decompose the other by BPS algorithm and find maximal atoms and minimal clique separators by restricting the product.

else

Step 2. Find all maximal atoms in $G$ and $H$ using BPS decomposition and let $S$ and $T$ be the corresponding set of atoms in $G$ and $H$, respectively.

Step 3. Remove all maximal atoms which are not cliques from $S$ and $T$ and let $S^{\prime}$ and $T^{\prime}$ be the set of maximal atoms in $G$ and $H$, respectively, obtained like this.

$\left.Q_{H}^{p}\right)$.

Step 4. For any two atoms $Q_{G}$ in $S^{\prime}$ and $Q_{H}$ in $T^{\prime}$, find $\left(Q_{G} \otimes Q_{H}\right)-\left(Q_{G}^{p} \otimes\right.$ 
Stop.

Correctness of the algorithm follows from Theorem 4.8. If $G$ has $n_{1}$ vertices and $m_{1}$ edges and $H$ has $n_{2}$ vertices and $m_{2}$ edges, the complexity of finding the clique minimal separators directly by BPS decomposition algorithm is now $O\left(n_{1} n_{2}\left(n_{1} m_{2}+n_{2} m_{1}+m_{1} m_{2}\right)\right)$. To check whether a maximal atom is a clique, has complexity $O\left(n_{1}^{2}\right)+O\left(n_{2}^{2}\right)$, and to find all pendant clique vertices has complexity $O\left(n_{1}^{2}\right)+O\left(n_{2}^{2}\right)$. So the total complexity is $O\left(n_{1} m_{1}+n_{2} m_{2}+n_{1}^{2}+n_{2}^{2}\right)$, which is much better again.

Acknowledgments. Work supported by the Ministry of Science of Slovenia and by the Ministry of Science and Technology of India under the bilateral India-Slovenia grants BI-IN/10-12-001 and INT/SLOVENIA-P-17/2009, respectively.

\section{REFERENCES}

1. B. S. Anand, M. Changat, S. Klavžar, I. Peterin: Convex sets in Lexicographic products of graphs. Graphs Combin, 28 (2012), 77-84.

2. K. Balakrishnan, M. Changat: Hull numbers of Path Convexities on Graphs. In: Convexity in Discrete Structures (M. Changat, S. Klavžar, H.M. Mulder, A. Vijayakumar, eds.), Lecture Notes Ser. 5, Ramanujan Math. Soc. (2008), 11-23.

3. T. Bartnicki, B. Brešar, J. Grytczuk, M. Kovše, Z. Miechowicz, I. Peterin: Game chromatic number of Cartesian product graphs. Electron. J. Combin., 15 (2008), R72.

4. A. Berry, R. Pogorelcnik, G. Simonet: An Introduction to Clique Minimal Separator decomposition. Algorithms, 3 (2010), 197-215.

5. B. BREŠAR, S. ŠPACAPAN: On the connectivity of the direct product of graphs. Australasian J. Combin., 41 (2008), 45-56.

6. G. DiRAC: On rigid circuit graphs. Abh. Math. Sem. Univ. Hamburg, 25 (1961), 71-76.

7. H. G. Leimer: Optimal decomposition by clique separators. Discrete Math., 113 (1993), 99-123.

8. M. Changat, H. M. Mulder, G. Sierksma: Convexities Related to Path Properties on Graphs. Discrete Math., 290 (2005), 117-131.

9. P. Duchet: Convex sets in graphs II, Minimal path convexity. J. Combin. Theory Ser. B., 44 (1988), 307-316.

10. P. Dorbec, M. Mollard, S. Klavžar, S. Špacapan: Power domination in product graphs. SIAM J. Discrete Math., 22 (2008), 554-567.

11. R. Hammack, W. Imrich, S. Klavžar: Handbook of Product Graphs, Second Edition. CRC Press, Boca Raton, FL, 2011.

12. P. ILLE: A proof of a conjecture of Sabidussi on graphs idempotent under the lexicographic product. Discrete Math., 309 (2009), 3518-3522.

13. R. J. Nowakowski, K. Seyffarth: Small cycle double covers of products. I. Lexicographic product with paths and cycles. J. Graph Theory, 57 (2008), 99-123. 
14. S. ŠpaCAPAN: Connectivity of Cartesian product of graphs. Appl. Math. Lett., 21 (2008), 682-685.

15. S. ŠPACAPAn: Connectivity of strong product of graphs. Graphs Combin., 26 (2010), 457-467.

16. R. E. TARJan: Decomposition by clique separators. Discrete Math., 55 (1985), 221232 .

17. P. M. Weichsel: The Kronecker product of graphs. Proc. Amer. Math. Soc., 13 (1962), 47-52.

18. X. ZHu: Game coloring the Cartesian product of graphs. J. Graph Theory, 59 (2008), 261-278.

Department of Futures Studies,

University of Kerala,

(Received October 4, 2011)

Trivandrum-695034

(Revised December 28, 2011)

India

E-mails: bijos_anand@yahoo.com

mchangat@gmail.com

Department of Computer Applications,

Cochin University of Science and Technology,

Cochin-22

India

E-mail: bkannan@cusat.ac.in

Institute of Mathematics and Physics,

Faculty of Electrical Engineering and Computer Science,

University of Maribor,

Smetanova 17, 2000 Maribor

Slovenia

E-mail: iztok.peterin@uni-mb.si 